\title{
Increased insulin secretory capacity but decreased insulin sensitivity after correction of iron overload by phlebotomy in hereditary haemochromatosis
}

\author{
D. Abraham • J. Rogers • P. Gault • J. P. Kushner • \\ D. A. McClain
}

Received: 30 June 2006 / Accepted: 31 July 2006 / Published online: 22 September 2006

(C) Springer-Verlag 2006

\begin{abstract}
Aims/hypothesis We recently demonstrated that humans with hereditary haemochromatosis have decreased insulin secretory capacity with a compensatory increase in insulin sensitivity. We therefore determined how these measures change after correction of tissue iron overload.

Subjects and methods Five non-diabetic subjects who had been studied previously at the time of initial diagnosis by means of the OGTT and frequently sampled intravenous glucose tolerance tests (FSIVGTT) underwent phlebotomy to normalise their serum ferritin. After normalisation of ferritin they were studied again ( $33 \pm 4$ months after the initial studies) by OGTT and FSIVGTT.

Results Normalisation of tissue iron stores resulted in an average 1.8-fold increase in the integrated area under the insulin curve during OGTT $(p<0.0001)$, but no significant change in the area under the glucose curve $(10 \%$ decrease, $p=0.32$ ). After phlebotomy, there was a 2.2-fold increase in insulin secretory capacity as determined by FSIVGTT (acute insulin response to glucose [AIRg], $p<0.02$ ) but a concomitant $70 \%$ fall in insulin sensitivity ( $\mathrm{Si}, p<0.05$ ).

D. Abraham · J. Rogers · P. Gault · J. P. Kushner • D. A. McClain Department of Internal Medicine,

University of Utah School of Medicine,

Salt Lake City, UT, USA

D. A. McClain

Salt Lake City Veterans Administration Health Care System,

Salt Lake City, UT, USA

D. A. McClain $(\bowtie)$

Division of Endocrinology, Metabolism and Diabetes,

University of Utah School of Medicine,

30 N. 2030 East,

Salt Lake City, UT 84132, USA

e-mail: donald.mcclain@hsc.utah.edu
\end{abstract}

The disposition index $(\mathrm{AIRg} \times \mathrm{Si})$ was unchanged $(5 \%$ increase, $p=0.90)$. BMI and fasting glucose were unchanged. At the time of diagnosis of haemochromatosis, four of the subjects had IGT. After normalisation of ferritin, two achieved NGT and two remained with IGT, despite 2.5and 3.7-fold increases in insulin secretory capacity.

Conclusions/interpretation Insulin secretory capacity improves after normalisation of iron stores in subjects with hereditary haemochromatosis. Glucose tolerance status improves incompletely because of decreased insulin sensitivity after phlebotomy. We conclude that tissue iron levels are an important determinant of insulin secretion and insulin action.

Keywords Diabetes $\cdot$ Haemochromatosis $\cdot$ Impaired glucose tolerance $\cdot$ Insulin resistance $\cdot$ Insulin secretion
Abbreviations
AIRg acute insulin response to glucose
FSIVGTT frequently sampled intravenous glucose tolerance test
$\mathrm{Si} \quad$ insulin sensitivity

\section{Introduction}

Hereditary haemochromatosis is transmitted as an autosomal recessive trait and occurs in approximately $0.5 \%$ of Europids of Northern European descent [1, 2]. Most patients with haemochromatosis are homozygous for a single nucleotide substitution (C282Y) in the haemochromatosis gene (HFE) [3]. Normal HFE expression is required for the regulation of hepcidin, a small hepatic peptide whose expression is induced by increased 
hepatic iron and inflammation [4]. Failure to induce hepcidin in haemochromatosis results in unregulated entry of iron into the circulation from the gastrointestinal tract and macrophages [5].

There is controversy about the frequency of morbidity that accompanies haemochromatosis. In relatively small clinical studies, the prevalence of diabetes in haemochromatosis has been found to be in the range of $7-40 \%[6,7]$. We recently reported a significantly increased prevalence of diabetes (23\%) and IGT (30\%) in adult subjects with haemochromatosis compared with matched controls $(0 \%$ diabetes and 14\% IGT) [8]. These rates of diabetes agreed well with the prevalence of diabetes $(26 \%)$ assessed by chart review of more than 200 subjects with haemochromatosis who had previously been studied by our group. Subjects with haemochromatosis and controls had similar insulin secretory capacity (acute insulin response to glucose [AIRg]) and insulin sensitivity ( $\mathrm{Si}$ ), as assessed by frequently sampled intravenous glucose tolerance testing (FSIVGTT). Haemochromatosis subjects with IGT exhibited a $68 \%$ decrease in AIRg compared with those with NGT. They were not insulin-resistant, instead exhibiting a $62 \%$ increase in $\mathrm{Si}$. The subjects with diabetes were overweight $(14 \%)$ or obese $(86 \%)$, suggesting the possibility that secondary insulin resistance could not be compensated because of decreased insulin secretory capacity. The phenotype of these subjects was mirrored in a mouse model of hereditary haemochromatosis with targeted deletion of $H f e$ or replacement of deleted wild-type Hfe with the cognate human mutation $\mathrm{C} 282 \mathrm{Y}$ [9]. These mice exhibited decreased insulin secretory capacity secondary to oxidative stress, decreased glucose-stimulated insulin secretion and beta cell apoptosis. Like the humans with haemochromatosis, the mice also had increased $\mathrm{Si}$ and they did not develop diabetes on a normal chow diet.

We therefore sought to determine the response of these subjects to phlebotomy and normalisation of tissue iron stores. We report that insulin secretory capacity increased significantly, but a parallel decrease in $\mathrm{Si}$ resulted in incomplete resolution of abnormalities in glucose tolerance.

\section{Subjects and methods}

Subjects

The study participants were recruited from all consecutive referrals to the Hemochromatosis Research Clinic at the University of Utah School of Medicine from 2000 to the present. All of these referred patients agreed to serve in the study and informed consent was obtained from those who agreed. HFE genotyping was performed using allele-specific PCR primers as previously described [8].
Five non-diabetic subjects who underwent study at the time of diagnosis agreed to repeat studies after normalisation of iron stores, as determined by serum ferritin, although one later declined the intravenous glucose tolerance test. The characteristics of this study population are shown in Table 1. All subjects' ferritin values were above normal before phlebotomy and were normalised by phlebotomy, although the change was not statistically significant because of the wide range of initial ferritin values (1230-14300 pmol/1). The average interval between the pre- and post-phlebotomy studies was $33 \pm 4$ (range 18-41) months. The average interval from normalisation of serum ferritin to the postphlebotomy study was $20 \pm 5$ (range 7-36) months. The study was approved by the University of Utah Institutional Review Board and the Advisory Committee to the General Clinical Research Center.

\section{Clinical studies}

After a 12-h overnight fast, an intravenous catheter was placed and subjects received an OGTT using a 75-g oral glucose load. Glucose and insulin values were determined at $0,30,60,90$ and $120 \mathrm{~min}$. AUCs were determined assuming linear changes between time points. Glucose tolerance status was defined according to WHO criteria [10]. Insulin was assayed by radioimmunoassay using a kit that has $20 \%$ cross-reactivity with proinsulin (Diagnostic Products, Los Angeles, CA, USA). The next morning, also after a 12-h fast, the subjects underwent an intravenous glucose tolerance test [11]. The subjects were given a $300 \mathrm{mg} / \mathrm{kg}$ glucose load over $1 \mathrm{~min}$; blood samples for insulin and glucose determination were collected at intervals of $1 \mathrm{~min}$ for $6 \mathrm{~min}$, then at 2-min intervals until $18 \mathrm{~min}$. At $20 \mathrm{~min}, 0.03 \mathrm{U} / \mathrm{kg}$ of regular insulin was infused over a 1-min interval. Blood was then drawn at 1-min intervals for $4 \mathrm{~min}$, then every $10 \mathrm{~min}$ until a total of $180 \mathrm{~min}$ had elapsed. Results were analysed using Minmod software [12] to determine AIRg, a measure of insulin secretory capacity, and $\mathrm{Si}$.

Table 1 Characteristics of the study population $(n=5)$

\begin{tabular}{lll}
\hline & Before phlebotomy & After phlebotomy \\
\hline Age (years) & $49.0 \pm 4.6$ & $50.8 \pm 4.9$ \\
Genotype & $100 \% \mathrm{C} 282 \mathrm{Y} / \mathrm{C} 282 \mathrm{Y}$ & - \\
Sex & $\begin{array}{l}\text { Two female, three } \\
\text { male }\end{array}$ & - \\
BMI $\left(\mathrm{kg} / \mathrm{m}^{2}\right)$ & $24.7 \pm 2.1$ & $24.3 \pm 1.5$ \\
Ferritin $(\mathrm{pmol} / \mathrm{l})$ & $4955 \pm 2386$ & $162 \pm 11$ \\
Glucose tolerance & NGT $n=1$, IGT $n=4$ & NGT $n=3$, IGT $n=2$ \\
$\quad$ status & & $4.98 \pm 0.08$ \\
Fasting glucose & $4.87 \pm 0.12$ & \\
$(\mathrm{mmol} / \mathrm{l})$ & & \\
\hline
\end{tabular}




\section{Statistics}

Statistical analyses were performed using SPSS software (SPSS, Chicago, IL, USA). Pre- and post-phlebotomy variables were analysed by paired $t$ test.

\section{Results}

Oral glucose tolerance testing

Fasting glucose values were unchanged after phlebotomy (Table 1). The results of OGTT are shown in Fig. 1. Although two of the subjects who were originally glucoseintolerant exhibited normal glucose tolerance after phlebotomy, two others remained glucose-intolerant and there was no significant change in the average AUC for serum glucose. The insulin AUC, however, uniformly and significantly increased by an average of 1.8 -fold $(p<0.0001)$.

Changes in insulin secretion and insulin sensitivity assessed FSIVGTT

The OGTT data, namely an increase in insulin levels without a comparable decrease in glucose excursions, suggested increased insulin secretory capacity but decreased insulin sensitivity as a result of phlebotomy. We therefore performed FSIVGTT to better quantify these variables. Testing confirmed a significant, 2.2-fold increase in insulin secretion (Fig. 2a; AIRg, $p<0.02$ ) and a $70 \%$ decrease in insulin sensitivity (Fig. $2 \mathrm{~b} ; \mathrm{Si}, p<0.05$ ). The disposition index, the product of AIRg and $\mathrm{Si}$, showed a variable response to phlebotomy, with no average change (Fig. 2c) but large variance among the subjects. The two subjects who remained glucose-intolerant after phlebotomy did so despite 2.5- and 3.7-fold increases in AIRg; they exhibited 75 and $76 \%$ decreases in Si respectively, despite no change in BMI.

\section{Discussion}

Our previously published results demonstrate that hereditary haemochromatosis secondary to mutations in the $H F E$ gene is accompanied by a significant prevalence of abnormalities in glucose homeostasis [8]. A substantial fraction of subjects, however, also had NGT, suggesting that there exist other genetic or environmental modifiers of the phenotype. At the stage of IGT, the primary abnormality appeared to be a decrease in insulin secretory capacity, which in a mouse model is associated with decreased beta cell mass and increased oxidative stress and apoptosis in the beta cells [9]. In both humans and in the mouse model, the loss of insulin secretory capacity was accompanied by increased insulin sensitivity. Thus, the mice with haemochromatosis did not develop diabetes on a normal chow diet, and in humans diabetes only occurred in the subpopulation that was overweight or obese $[8,9]$. This suggests that secondary insulin resistance, resulting not

a

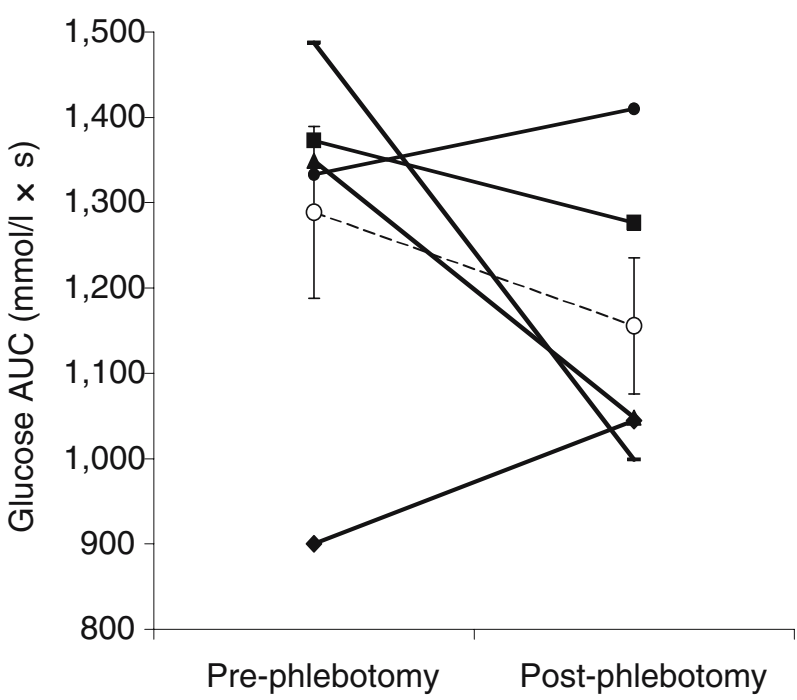

b

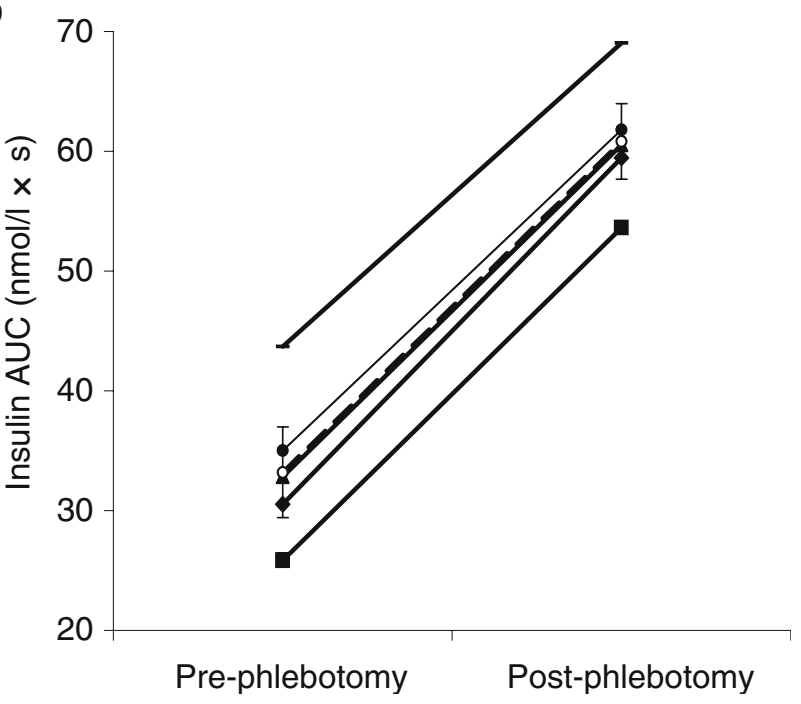

Fig. 1 Integrated areas under the serum glucose (a) and insulin (b) curves (AUC) after oral glucose tolerance testing. Values for each subject before and after phlebotomy are shown as solid lines with closed symbols, and the group average as the dashed line and open circles $( \pm \mathrm{SE})$. Individual subjects are identified in this and subsequent figures with the different closed symbols: Subject 1, closed squares, IGT before and after phlebotomy; Subject 2, closed circles, IGT before and after phlebotomy; Subject 3, closed triangles, IGT before and NGT after phlebotomy; Subject 4, closed diamonds, NGT before and after phlebotomy; Subject 5, horizontal bar, IGT before and NGT after phlebotomy. $p=0.32$ for the $10 \%$ decrease in area under the glucose curve after phlebotomy; $p<0.0001$ for the increase in insulin values 


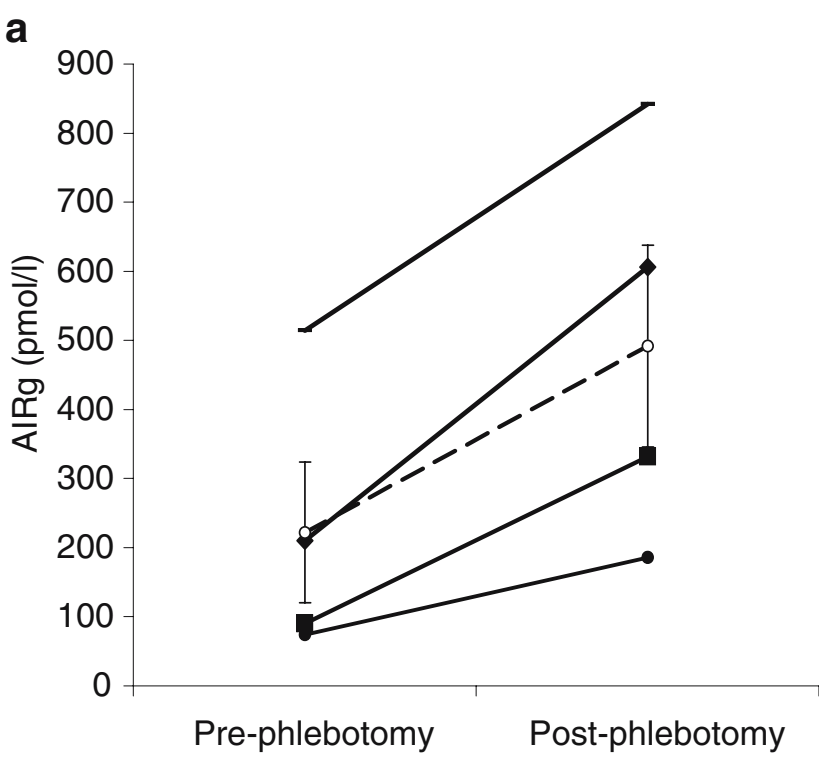

b
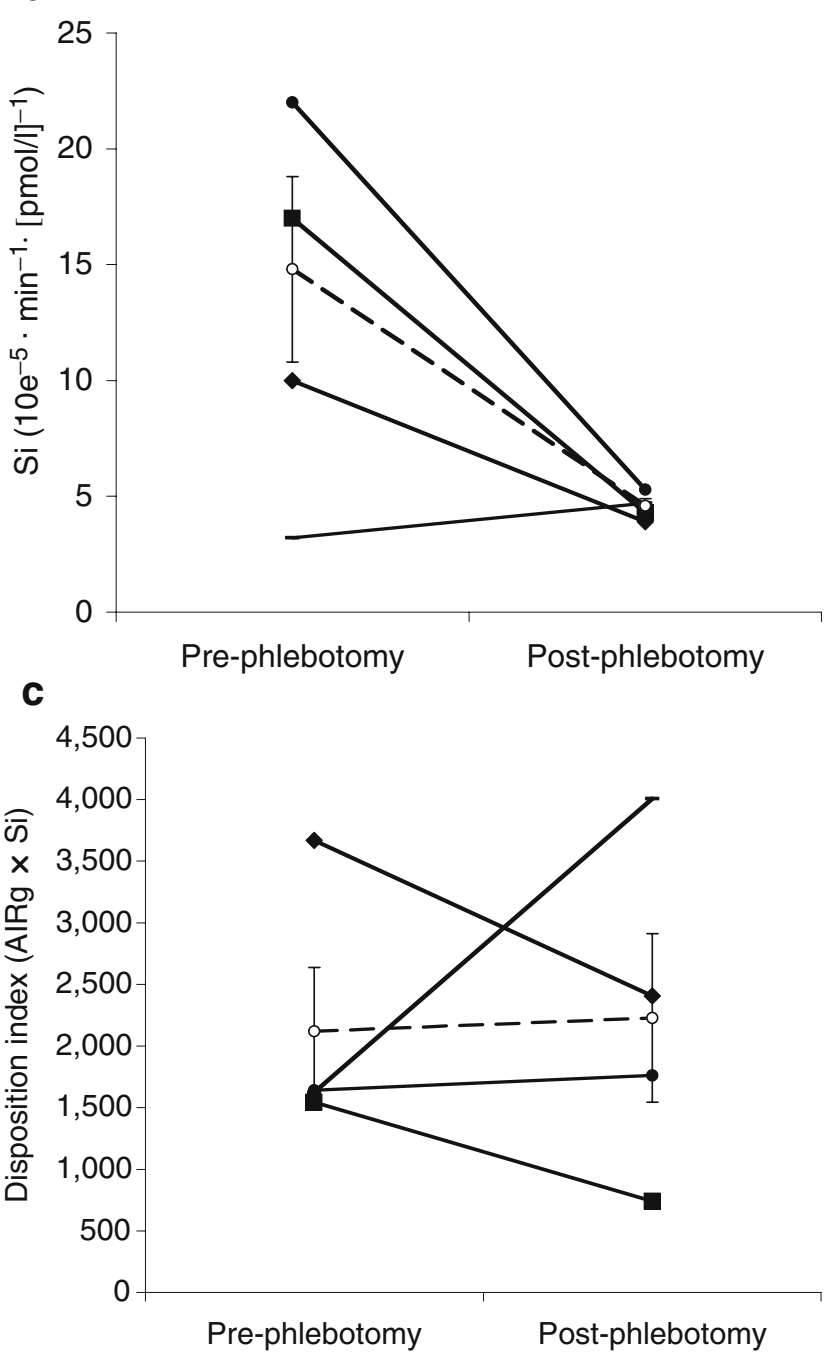

Fig. 2 Insulin secretion, $\mathrm{Si}$ and glucose disposition indices in subjects with haemochromatosis before and after phlebotomy determined by the FSIVGTT. Values for each subject before and after phlebotomy are shown as solid lines and closed circles and the group average as the dashed line and open circles $( \pm \mathrm{SE})$. Data were analysed by the Bergman minimal model using Minmod software [12]. Individual subjects are identified with the following symbols: Subject 1, closed squares, IGT before and after phlebotomy; Subject 2, closed circles, IGT before and after phlebotomy; Subject 3 depicted in Fig. 1 declined the FSIVGTT; Subject 4, closed diamonds, NGT before and after phlebotomy; Subject 5, horizontal bar, IGT before and NGT after phlebotomy. a Acute insulin response to glucose (AIRg, $p<0.02$ for the difference after phlebotomy). b Si, $p<0.05$ for the difference after phlebotomy. c Disposition index (DI, $p=0.9$ )

from the haemochromatosis itself but from obesity and/or other factors, such as cirrhosis [8], is also a factor in the development of diabetes with haemochromatosis.

We therefore sought in the present study to determine whether this phenotype of decreased insulin secretory capacity with increased insulin sensitivity would be reversed by phlebotomy and normalisation of tissue iron levels. Five subjects, four of whom had IGT, were therefore restudied by both oral and intravenous glucose tolerance testing an average of $20 \pm 5$ months after normalisation of serum ferritin. Both the area under the insulin curve during OGTT and the AIRg during FSIVGTT increased significantly after phlebotomy. However, the area under the glucose curve did not change significantly, and there was a decrease in $\mathrm{Si}$ as determined by FSIVGTT, such that the glucose tolerance status of two of the subjects with IGT did not improve despite 2.5- and 3.7-fold increases in AIRg.

The results suggest that there are complex inter-relationships among iron status, insulin secretion and insulin sensitivity. It might have been predicted, for example, that individuals with IGT based solely on an insufficient insulin secretory response would regain normal glucose tolerance with a 2- to 4-fold increase in insulin secretory capacity, but this did not occur. The results are more consistent with one or both of two possibilities: that insulin secretion rates control insulin sensitivity, and/or that iron is an independent factor in regulating both insulin secretion and insulin sensitivity. Regarding the former hypothesis, it is most often thought that insulin secretion responds to insulin sensitivity rather than vice versa. For example, the hyperinsulinaemia of obesity and early type 2 diabetes is thought to be a compensatory mechanism for insulin resistance [13]. However, there are also examples in human pathology and a wide variety of experimental models in which insulin secretion determines insulin sensitivity. That is, low levels of insulin secretion can be compensated to maintain NGT, and conversely primary hyperinsulinaemia can induce insulin resistance [14-18]. However, such a relationship between insulin secretion and insulin sensitivity does not explain the lack of improvement of IGT despite increased 
insulin secretion. Analysis of mice with iron overload suggests instead that iron itself plays a central role in the regulation of metabolism and insulin sensitivity. We have noted, for example, increased glucose disposal rates, activation of AMP-dependent kinase (AMPK) and resistance to diet-induced obesity with increased thermogenesis in iron-overloaded mice (DA McClain and RC Cooksey, unpublished results). In lower organisms, especially in the yeast Saccharomyces cerevisiae, the connections between iron and metabolism are well established. For example, Snf1 (AMP-dependent kinase) is involved in signalling the shift from oxidative to fermentative metabolism and also controls the induction of iron transport genes [19]. In humans, the mechanisms for the regulation of insulin action and glucose homeostasis by iron are unknown, however.

The present data do not support insulin resistance as being a primary consequence of iron overload. Previous studies have suggested that both insulin deficiency and insulin resistance are contributing factors in the diabetes of haemochromatosis [20-22]. Some of this work is difficult to interpret because subjects with established diabetes were studied, in which case the attendant hyperglycaemia may itself have resulted in insulin resistance and insulin secretory abnormalities [23]. Furthermore, the studies most clearly demonstrating iron effects on insulin sensitivity in patients without diabetes are those of subjects with transfusional or idiopathic iron overload, and not of subjects with haemochromatosis $[21,24]$. The tissue distribution of iron may differ significantly between dietary iron overload and haemochromatosis, and lead to different phenotypes of fuel homeostasis. For example, in dietary iron overload there is iron accumulation in macrophages, which is not the case in haemochromatosis because macrophages express ferroportin, and the lack of downregulation of ferroportin due to the deficiency of hepcidin allows free egress of iron from these cells [25]. This may explain why increased iron stores in the absence of haemochromatosis are associated epidemiologically with the development of typical type 2 diabetes rather than the insulin-sensitive phenotype reported here [26-29].

In summary, we report that after phlebotomy therapy to reduce iron overload in hereditary haemochromatosis, insulin secretory capacity improves significantly but insulin sensitivity decreases. The net result of these changes is that glucose intolerance may either resolve or remain in individual subjects. The results suggest that iron, more specifically the iron status of specific tissues, may play a key role in determining insulin secretion, insulin action and metabolic regulation.

Acknowledgements This work was supported by the Research Service of the Veterans Administration, the National Institutes of Health (RO1 DK059512), the Huntsman General Clinical Research
Center (MO1 RR000064), and the Ben B. and Iris M. Margolis Foundation.

Duality of interest The authors have no commercial relationships or duality of interest relevant to this manuscript.

\section{References}

1. Edwards C (1999) Hemochromatosis. In: Lee GR Foerster J, Lukens J, Paraskevas F, Greer JP, Rodgers GM (eds) Wintrobe's clinical hematology. Williams \& Wilkins, Baltimore, pp 10561070

2. Bothwell TH, Chariton RW, Motulsky AG (1995) Hemochromatosis. In: Scriver CR Beaudet AL, Sly WS, Valle D (eds) The metabolic and molecular bases of inherited disease. McGraw-Hill, New York, pp 2237-2269

3. Feder JN, Gnirke A, Thomas W et al (1996) A novel MHC class I-like gene is mutated in patients with hereditary haemochromatosis. Nat Genet 13:399-408

4. Nemeth E, Roetto A, Garozzo G, Ganz T, Camaschella C (2004) Hepcidin is decreased in TFR2-hemochromatosis. Blood 105:1803-1806

5. Nemeth E, Tuttle MS, Powelson J et al (2004) Hepcidin regulates cellular iron efflux by binding to ferroportin and inducing its internalization. Science 306:2090-2093

6. Buysschaert M, Paris I, Selvais P, Hermans MP (1997) Clinical aspects of diabetes secondary to idiopathic haemochromatosis in French-speaking Belgium. Diabetes Metab 23:308-313

7. Moirand R, Adams PC, Bicheler V, Brissot P, Deugnier Y (1997) Clinical features of genetic hemochromatosis in women compared with men. Ann Intern Med 127:105-110

8. McClain DA, Abraham D, Rogers J et al (2006) High prevalence of abnormal glucose homeostasis secondary to decreased insulin secretion in individuals with hereditary haemochromatosis. Diabetologia 49:1661-1669

9. Cooksey RC, Jouihan HA, Ajioka RS et al (2004) Oxidative stress, beta-cell apoptosis, and decreased insulin secretory capacity in mouse models of hemochromatosis. Endocrinology 145:5305-5312

10. Alberti KG, Zimmet PZ (1998) Definition, diagnosis and classification of diabetes mellitus and its complications. Part 1: diagnosis and classification of diabetes mellitus provisional report of a WHO consultation. Diabet Med 15:539-553

11. Saad MF, Steil GM, Riad-Gabriel M et al (1997) Method of insulin administration has no effect on insulin sensitivity estimates from the insulin-modified minimal model protocol. Diabetes 46:2044-2048

12. Boston RC, Stefanovski D, Moate PJ, Sumner AE, Watanabe RM, Bergman RN (2003) MINMOD Millennium: a computer program to calculate glucose effectiveness and insulin sensitivity from the frequently sampled intravenous glucose tolerance test. Diabetes Technol Ther 5:1003-1015

13. Kahn BB (1998) Type 2 diabetes: when insulin secretion fails to compensate for insulin resistance. Cell 92:593-596

14. Seaquist ER, Pyzdrowski K, Moran A, Teuscher AU, Robertson RP (1994) Insulin-mediated and glucose-mediated glucose uptake following hemipancreatectomy in healthy human donors. Diabetologia 37:1036-1043

15. Rizza RA, Mandarino LJ, Genest J, Baker BA, Gerich JE (1985) Production of insulin resistance by hyperinsulinaemia in man. Diabetologia 28:70-75 
16. Del Prato S, Riccio A, Vigili de Kreutzenberg S et al (1993) Mechanisms of fasting hypoglycemia and concomitant insulin resistance in insulinoma patients. Metabolism 42:24-29

17. Miles PD, Li S, Hart M et al (1998) Mechanisms of insulin resistance in experimental hyperinsulinemic dogs. J Clin Invest 101:202-211

18. Koopmans SJ, Kushwaha RS, DeFronzo RA (1999) Chronic physiologic hyperinsulinemia impairs suppression of plasma free fatty acids and increases de novo lipogenesis but does not cause dyslipidemia in conscious normal rats. Metabolism 48:330-337

19. Haurie V, Boucherie H, Sagliocco F (2003) The Snfl protein kinase controls the induction of genes of the iron uptake pathway at the diauxic shift in Saccharomyces cerevisiae. J Biol Chem 278:45391-45396

20. Hramiak IM, Finegood DT, Adams PC (1997) Factors affecting glucose tolerance in hereditary hemochromatosis. Clin Invest Med 20:110-118

21. Merkel PA, Simonson DC, Amiel SA et al (1988) Insulin resistance and hyperinsulinemia in patients with thalassemia major treated by hypertransfusion. N Engl J Med 318:809-814

22. Mendler MH, Turlin B, Moirand R et al (1999) Insulin resistanceassociated hepatic iron overload. Gastroenterology 117:11551163
23. Rossetti L, Giaccari A, DeFronzo RA (1990) Glucose toxicity. Diabetes Care 13:610-630

24. Bugianesi E, Manzini P, D'Antico S et al (2004) Relative contribution of iron burden, HFE mutations, and insulin resistance to fibrosis in nonalcoholic fatty liver. Hepatology 39:179-187

25. Viatte L, Nicolas G, Lou DQ et al (2006) Chronic hepcidin induction causes hyposideremia and alters the pattern of cellular iron accumulation in hemochromatotic mice. Blood 107:29522958

26. Fleming DJ, Jacques PF, Tucker KL et al (2001) Iron status of the free-living, elderly Framingham Heart Study cohort: an ironreplete population with a high prevalence of elevated iron stores. Am J Clin Nutr 73:638-646

27. Fernandez-Real JM, Lopez-Bermejo A, Ricart W (2002) Crosstalk between iron metabolism and diabetes. Diabetes 51:23482354

28. Ford ES, Cogswell ME (1999) Diabetes and serum ferritin concentration among U.S. adults. Diabetes Care 22:1978-1983

29. Fleming DJ, Tucker KL, Jacques PF, Dallal GE, Wilson PW, Wood RJ (2002) Dietary factors associated with the risk of high iron stores in the elderly Framingham Heart Study cohort. Am J Clin Nutr 76:1375-1384 